\title{
Eighty years of research on super-heavy nuclei
}

\author{
Sigurd Hofmann ${ }^{1,2, a}$ \\ ${ }^{1}$ GSI Helmholtzzentrum für Schwerionenforschung, Planckstrasse 1, 64291 Darmstadt \\ ${ }^{2}$ Institut für Physik, Goethe-Universität Frankfurt, 60438 Frankfurt
}

\begin{abstract}
Professor Walter Greiner, our mentor, colleague, and friend, passed away in the age of eighty. During his lifetime, the search for elements beyond uranium started and elements up to the so far heaviest one with atomic number 118 were discovered. In this talk I will present a short history from early searches for 'trans-uraniums' up to the production and safe identification of shell-stabilized 'Super-Heavy Nuclei' (SHN). The nuclear shell model reveals that these nuclei should be located in a region with closed shells for the protons at $\mathrm{Z}=114,120$ or 126 and for the neutrons at $\mathrm{N}=184$. The outstanding aim of experimental investigations is the exploration of this region of spherical SHN. Systematic studies of heavy ion reactions for the synthesis of SHN revealed production cross-sections which reached values down to one picobarn and even below for the heaviest species. The systematics of measured cross-sections can be understood only on the basis of relatively high fission barriers as predicted for nuclei in and around the island of SHN. A key role in answering some of the open questions plays the synthesis of isotopes of element 120 . Attempts aiming for synthesizing this element at the velocity filter SHIP will be reported.
\end{abstract}

\section{Review and status of experiments}

Scientific attempts to synthesize new elements beyond uranium started in the 1930s, when the atomic model was established and the constituents of the atomic nucleus, protons and neutrons, were known. E. Fermi in Rome [1] and O. Hahn, L. Meitner, and F.W. Straßmann in Berlin [2] tried to use the nuclear reaction of neutron capture by uranium target nuclei and subsequent $\beta^{-}$decay for production of transuranium elements. Although first results were misinterpreted, the experiments opened a new area of research in nuclear physics. A chart of nuclei existing in 1935, the relevant year of this special session of the conference, is plotted on top of fig. 1.

The discovery of Hahn and Straßmann [3] in 1938 was that uranium, more accurate the odd-mass isotope ${ }^{235} \mathrm{U}$, breaks into two approximately equal parts after neutron capture. This new phenomenon of nuclear fission was described by Meitner and O.R. Frisch in 1939 using the charged liquid-drop model [4]. One year later, G.N. Flerov and K.A. Petrjak [5] detected that uranium, ${ }^{238}$ U, decays spontaneously by fission from its ground-state.

The first new elements beyond uranium were synthesized during the years of the Second World War in laboratories in the US. These were the elements from neptunium $(Z=93)$ to curium (96). In

ae-mail: S.Hofmann@gsi.de 


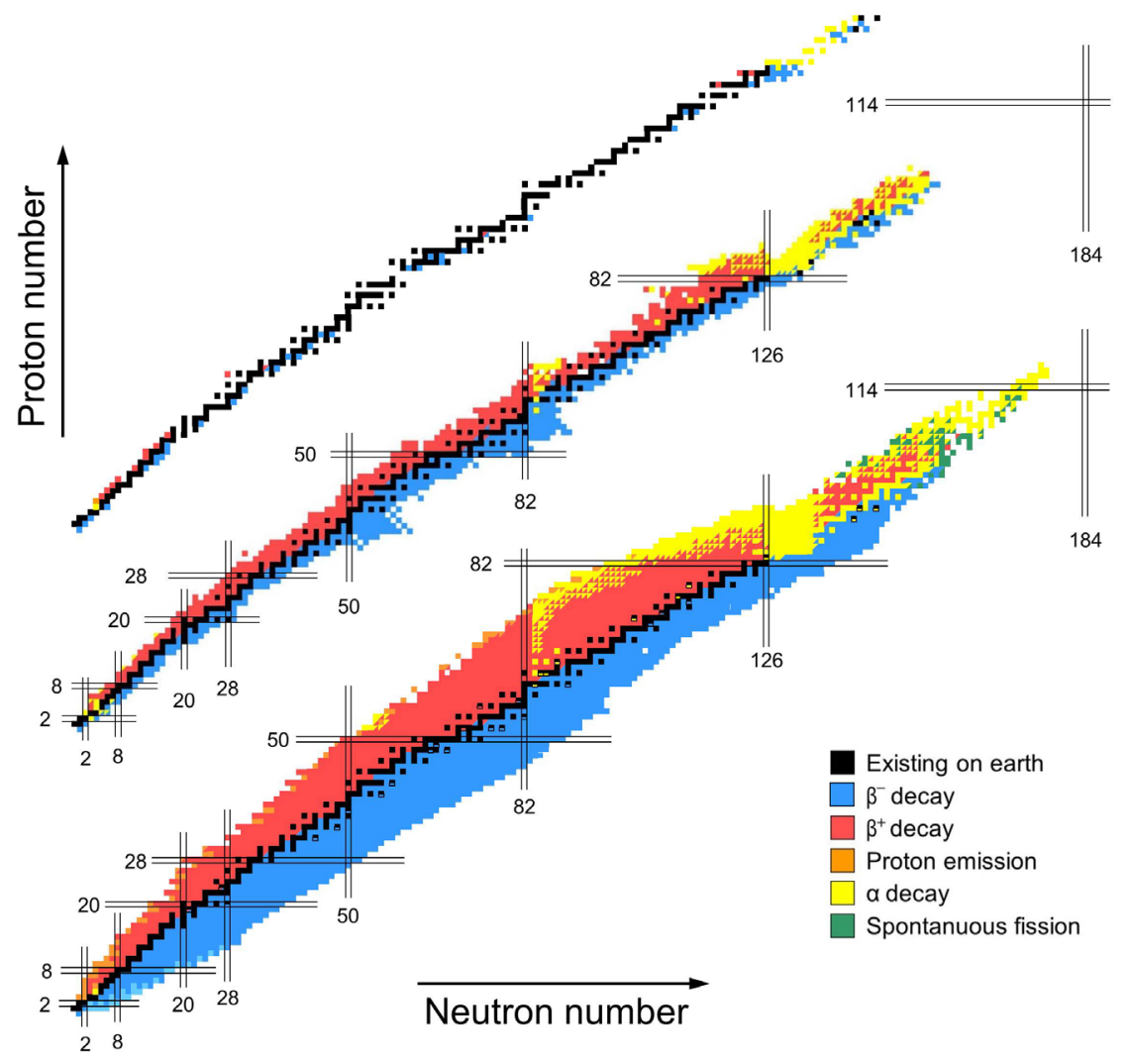

Figure 1. Three charts of nuclei from the years 1935, 1958, and 2015 demonstrating the advance in nuclear physics during 80 years of research. The nuclei known in 1935 were compiled by G. Fea working at the School of Nuclear Physics in Rome in collaboration with F. Rasetti and E. Segré [6]. The heaviest nucleus known at that time was ${ }^{238} \mathrm{U}$. Most of the stable isotopes have been identified using mass spectrograph's. Radioactive nuclei were known from the $\alpha$ decay of uranium and thorium isotopes and at the lower end of the chart from nuclear reactions of those $\alpha$ particles with low $\mathrm{Z}$ target nuclei. The arrangement of the original chart was changed to an $\mathrm{N}$ over $\mathrm{Z}$ plot and the isotopes were colored according to the convention of the 'Karlsruher Nuklidkarte' shown in the lower part, which was compiled and published for the first time in 1958 [7].

the years 1949-55 the elements from berkelium (97) to mendelevium (101) were also produced in the US.

The production processes were capture of fast neutrons from a reaction of ${ }^{2} \mathrm{H}$ with ${ }^{9} \mathrm{Be}$ by ${ }^{238} \mathrm{U}$ and subsequent $\beta^{-}$decay $\left({ }^{239} \mathrm{~Np}\right), \beta^{-}$decay of ${ }^{238} \mathrm{~Np}$ which was produced from ${ }^{238} \mathrm{U}$ in irradiations with ${ }^{2} \mathrm{H}\left({ }^{238} \mathrm{Pu}\right)$, slow neutron capture by ${ }^{240} \mathrm{Pu}$ produced from ${ }^{239} \mathrm{Pu}$ in a nuclear reactor and subsequent $\beta^{-}$ decay $\left({ }^{241} \mathrm{Am}\right)$, fusion using a ${ }^{4} \mathrm{He}$ beam from the 60 -inch cyclotron in Berkeley $\left({ }^{242} \mathrm{Cm},{ }^{243} \mathrm{Bk},{ }^{245} \mathrm{Cf}\right.$, ${ }^{256} \mathrm{Md}$ ), and rapid capture of 15 and 17 neutrons by ${ }^{238} \mathrm{U}$ in a thermonuclear explosion and subsequent $\beta^{-}$decays $\left({ }^{253} \mathrm{Es},{ }^{255} \mathrm{Fm}\right)$.

Chemical separation of these new elements was essential for the identification, as it was already for the discovery of nuclear fission, which was identified by the observation of barium in a chemically 
separated sample. In the region of heavy elements, these studies resulted in the concept of a second series of chemically similar elements, the actinides, starting at element 89 , actinium, besides the known lanthanides, both having unfilled f-electron shells. In 1951, G.T. Seaborg and E.M. McMillan received the Nobel Prize in Chemistry, "for their discoveries in the chemistry of the transuranium elements".

Limits of existence of nuclei were presented by J.A. Wheeler in a compilation of essays dedicated to Niels Bohr on the occasion of his 70th birthday [8] and at a conference 'On the peaceful uses of atomic energy' in Geneva in 1955 [9]. Solely based on the charged liquid-drop model, the results seemed reasonable, "to look for nuclei with a well defined existence with masses perhaps two or more times heavier than the heaviest nucleus now known, ${ }^{256} 100$ ". Whereas in [8] these nuclei were still named 'very heavy nuclei', the term 'superheavy nuclei', now usually abbreviated SHN, was used in [9] for the first time. Two years later, F.G. Werner and Wheeler published a paper with the title 'Superheavy Nuclei' [10], in which the properties of these nuclei were estimated in more detail but still disregarding shell effects.
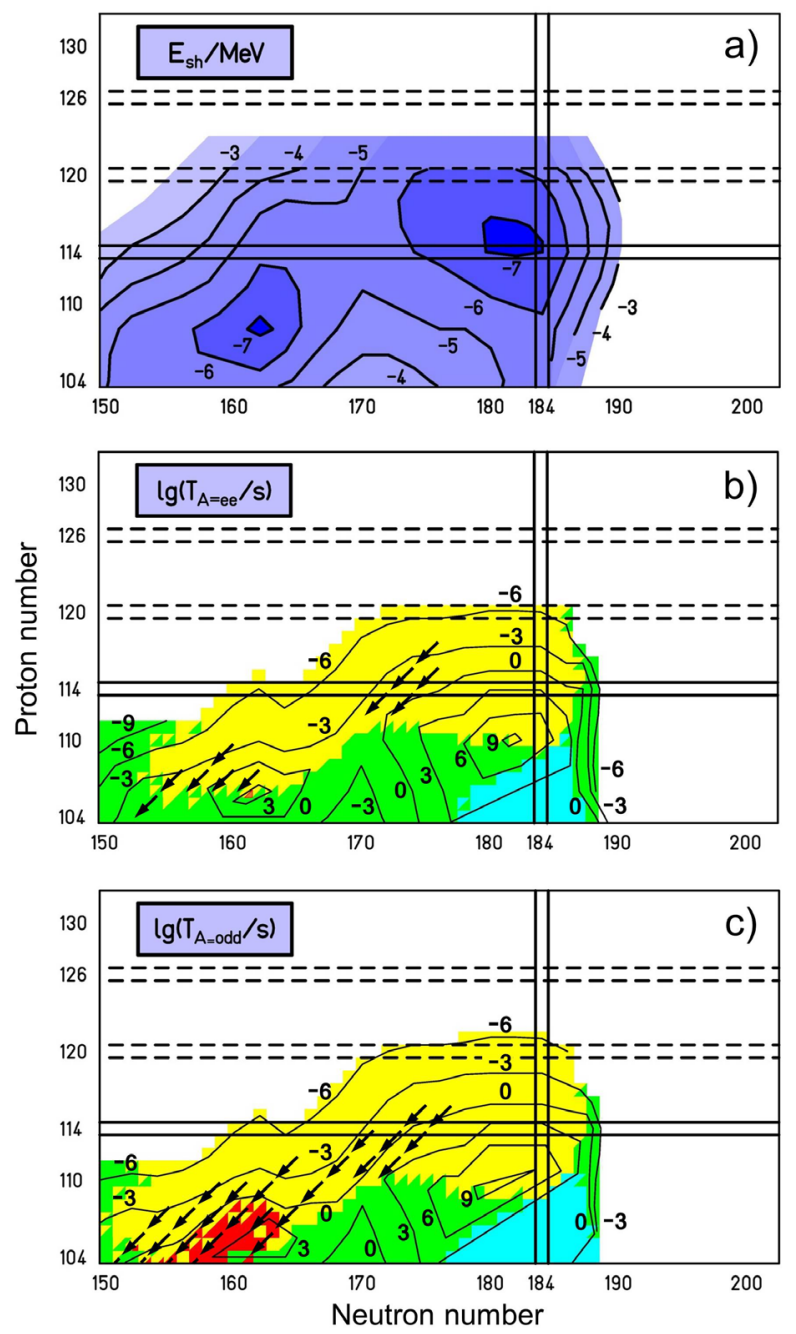

Figure 2. Shell-correction energies in $\mathrm{MeV}$ taken from $[17,18]$ (a) and dominating decay modes of even-even nuclei (b) and of even-odd nuclei (c). The dominating decay modes were determined from partial half-lives for $\alpha$ decay (yellow), $\beta^{+}$decay or electron capture (red), $\beta^{-}$decay (blue), and SF (green) calculated in [15-17]. Hindrance factors of 10 and 1000 were assumed for $\alpha$ decay and SF of even-odd nuclei, respectively. Arrows mark measured decay chains starting at the even element isotopes ${ }^{264} \mathrm{Hs},{ }^{270} \mathrm{Ds},{ }^{268} \mathrm{Hs},{ }^{270} \mathrm{Hs}$, ${ }^{294} 118$, and ${ }^{292} \mathrm{Lv}$ in (b) and at ${ }^{263} \mathrm{Hs},{ }^{269} \mathrm{Ds}$, ${ }^{271} \mathrm{Ds},{ }^{277} \mathrm{Cn},{ }^{271} \mathrm{Hs},{ }^{285} \mathrm{Fl},{ }^{291} \mathrm{Lv}$, and ${ }^{293} \mathrm{Lv}$ in (c). The $\alpha$-decay chains of even-even nuclei and most of the chains of even-odd nuclei end by $\mathrm{SF}$ in agreement with predictions. 
Also discussed at the Geneva conference was the problem of the binding of electrons in the strong electric field of such 'superheavy nuclei'. This question was brought up by D.I. Blokhintsev in the discussion of Wheeler's contribution [9]. Blokhintsev referred to the term $-Z^{2} \alpha^{2}$ as radicand in the Dirac equation, which causes that calculation of the binding energy of $\mathrm{K}$ electrons fails for pointlike nuclei. However, Wheeler replied that they have found "that for a finite size of the nucleus even with a nuclear charge of 170 , the $\mathrm{K}$ electron has a perfectly reasonable wave function and has a binding energy of about $1.85 \mathrm{~m}_{e} \mathrm{c}^{2}$ ".

This subject and related vacuum polarization and electron-positron pair creation in strong electric fields became later a major topic of theoretical studies at Greiner's Institute at University Frankfurt $[11,12]$ and of experimental work at GSI (Gesellschaft für Schwerionenforschung) in Darmstadt [13].

In 1948, the magic numbers were successfully explained by the nuclear shell model [14, 15], and an extrapolation into the region of the next doubly magic nuclei beyond ${ }^{208} \mathrm{~Pb}$ was thus undertaken. The numbers 126 for the protons, later changed to 114 [22], and 184 for the neutrons were predicted to be the next spherical shell closures.

The perspectives offered by the nuclear shell model for production of SHN and the need for developing more powerful accelerators for their synthesis in heavy ion reactions was a main motivation for upgrading existing facilities or for founding new laboratories. In expectation of broad research fields, the HILAC (Heavy Ion Linear Accelerator), later upgraded to the SuperHILAC, was built at LBNL (Lawrence Berkeley National Laboratory) in Berkeley in 1955, the U-300 and U-400 cyclotrons at FLNR (Flerov Laboratory of Nuclear Reactions) at JINR (Joint Institute for Nuclear Research) in Dubna in 1957 and 1978, respectively, the UNILAC (Universal Linear Accelerator) at GSI in 1969, and the RILAC (RIKEN variable-frequency Linear Accelerator) at the RIKEN Nishina Center in Saitama near Tokio in 1980.

Studies of the elements 100 to 106 were performed with the new cyclotron U-300 using fusion reactions with beams of ${ }^{12} \mathrm{C}$ to ${ }^{22} \mathrm{Ne}$. In recognition of this early work in Dubna, element 105 is now officially named 'dubnium'.

At approximately the same time, the experiments at the HILAC in Berkeley culminated in the synthesis of the new element 106. After careful and deliberate determination of well balanced discovery profiles by the International Unions of Pure and Applied Chemistry (IUPAC) and Physics (IUPAP), the names 'nobelium', 'lawrencium', and 'rutherfordium' are now officially accepted for the elements 102, 103, and 104, respectively, as well as 'seaborgium' for element 106.

In the middle of the 1960s, the concept of the macroscopic-microscopic (MM) model for calculating binding energies of nuclei also at large deformations was invented by V.M. Strutinsky [20]. Using this method a number of the measured phenomena could be naturally explained. In particular, it became possible to calculate the binding energy of a heavy fissioning nucleus as function of deformation and thus to determine the fission barrier. Partial fission half-lives were calculated using the so determined fission barrier. The calculations revealed the existence of so called 'islands of stability' far beyond the known nuclei [21-31].

Other important results which could be explained applying the Strutinsky method for calculation of the structure of the fission barrier are the fission isomers discovered by S.M. Polikanov et al. [32], which gain their stability from a second minimum in the fission barrier at large deformation, and the detection of the break of systematically long half-lives of $N=152$ isotones at element 104 by Yu.Ts. Oganessian et al. [33] due to the disappearance of a second hump in the fission barrier.

The calculation of ground-state shell correction energies (SCE) of the MM model revealed a minimum (maximum in terms of stability) not only for spherical SHN at Z $=114$ and $N=184$, but also for deformed nuclei at $\mathrm{Z}=108$ and $\mathrm{N}=162$ [26]. The two minima, both having SCE values of $-7 \mathrm{MeV}$, 
are clearly visible in fig. 2a. The figure shows SCE values taken from a calculation of A. Sobizcewski et al. [18] for a wide range of heavy and super-heavy nuclei.

The shift of SHN with lowest SCE values to the region slightly above the shell closure at $\mathrm{Z}=114$ and slightly below the shell closure at $\mathrm{N}=184$ is due to the low level density for the protons between 114 and 126 and for the neutrons between 164 and 184, see graphs 53 and 54 in [19]. The nuclei at Z $=108$ and $\mathrm{N}=162$ gain their stability from relatively high level densities below gaps of single particle levels for these nucleon numbers at deformations characterized by the deformation parameters $\beta_{2} \approx$ $0.22, \beta_{4} \approx-0.07$ [18]. However, these gaps between single particle levels of deformed nuclei do not result from shell closures in terms of the classical shell model for spherical nuclei.

The ridge of maximum SCE values between the two minima separates the region of heavy and super-heavy nuclei. Roughly, the borderline follows the line of constant mass number at $\mathrm{A}=280$. This definition of SHN is in agreement with definitions given in early calculations of the stability of SHN. However, it differs from the definition used by nuclear chemists nowadays, who define as super-heavy elements (SHE) the elements beyond the actinide series beginning with rutherfordium, element 104.

The calculation of spontaneous fission (SF) half-lives of SHN was still problematic. It depends sensitively from the size of the fission barrier, the inertia and for odd and odd-odd nuclei from nuclear structure effects which generate an increase of the fission barrier by the so called specialization energy. Predicted half-lives based on the Strutinsky model using various parameter sets differed by many orders of magnitude. Some of the half-lives approached the age of the universe, and attempts have been made to discover naturally occurring SHN [36-39]. Although the corresponding discoveries were announced from time to time, none of them could be substantiated after more detailed inspection.

Even the location of the closed shells for protons and neutrons turned out to be model dependent. Self-consistent Hartree-Fock-Bogoliubov calculations and relativistic mean field models [40-45] predict for spherical nuclei shells at $Z=114,120$, or 126 (indicated as dashed lines in fig. 2) and $N=172$ or 184. In terms of the shell model, the uncertainty in $Z$ and $N$ is due to the uncertain spin-orbit splitting of shells and subshells of high and low angular momentum, respectively, from 114 to 126 for the protons and from 164 to 184 for the neutrons.

The shortest half-lives which determine the decay mode are plotted in fig. $2 b$ for even-even nuclei and in fig. 2c for even-odd nuclei. For the odd nuclei partial $\alpha$ and SF half-lives calculated in [18] were multiplied by factors of 10 and 1000, respectively, thus making provisions for the odd particle hindrance factors. However, one has to keep in mind that, in particular, fission hindrance factors show a wide distribution from $10^{1}$ to $10^{5}$, which is mainly a result of the specific levels occupied by the odd nucleon $[9,46]$.

For even-even nuclei in fig. $2 b$, the two regions of deformed heavy nuclei near $\mathrm{N}=162$ and spherical SHN merge and form a region of $\alpha$ emitters surrounded by spontaneously fissioning nuclei. Alpha decay becomes the dominant decay mode beyond $\mathrm{Z}=110$ with continuously decreasing halflives. For nuclei at $\mathrm{N}=184$ and $\mathrm{Z}<110$ half-lives are determined by $\beta^{-}$decay. For even-odd nuclei, fig. 2c, the island character of $\alpha$ emitters disappears and for nuclei with neutron numbers 150 to 160 $\alpha$ decay prevails down to element 104 and beyond.

Longest total half-lives do not occur for nuclei having the most negative SCE values. Due to the short partial $\alpha$ half-lives there, the longest half-lives of SHN are predicted for nuclei near element 110 and neutron number 182 .

The set-ups used in physics experiments for the investigation of SHN are described in detail in review articles [47-53]. Cold and hot fusion reactions based on targets of lead or bismuth and isotopes of actinides, respectively, were used for the synthesis of heavy and super-heavy nuclei. These experiments resulted in the identification of the new elements 107 to 112 at the vacuum velocity filter SHIP 


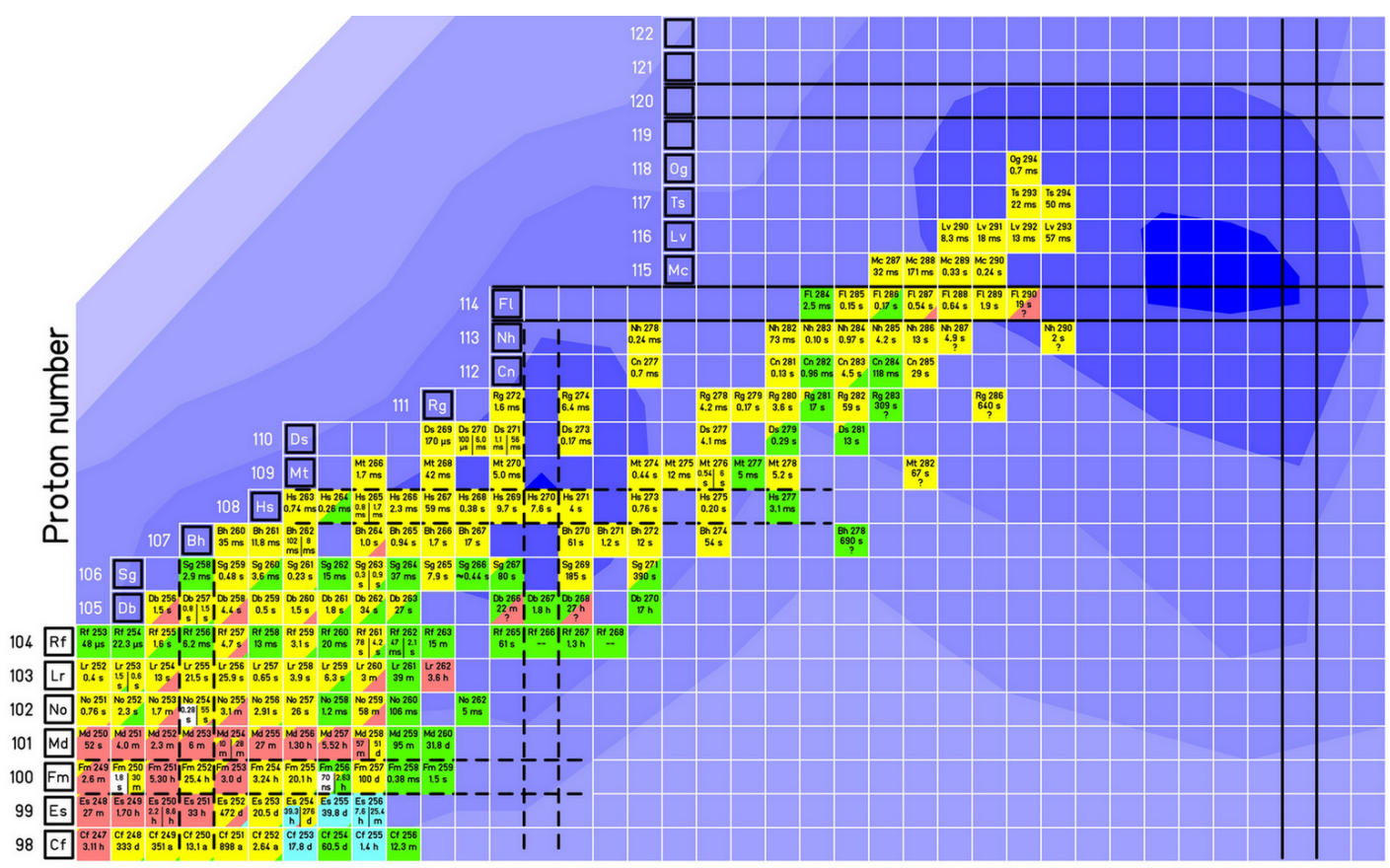

149150151152153154155156157158159160161162163164165166167168169170171172173174175176177178179180181182183184185186 Neutron number

Figure 3. Upper end of the chart of nuclei showing the presently (2017) known nuclei. For each known isotope the element name, mass number, and half-life are given. The elements from proton number 107 to 113 were discovered in fusion reactions after evaporation of one neutron from the compound nucleus (cold fusion reactions based on ${ }^{208} \mathrm{~Pb}$ and ${ }^{209} \mathrm{Bi}$ targets). The corresponding isotopes are located on the more neutron deficient side and cover the region of deformed heavy nuclei with the center at $\mathrm{Z}=108$ and $\mathrm{N}=162$. The more neutron rich isotopes of elements 112 and 113 were produced in reactions using a ${ }^{48} \mathrm{Ca}$ beam and targets of ${ }^{238} \mathrm{U}$ and ${ }^{237} \mathrm{~Np}$ and the new elements from 114 to 118 with targets of ${ }^{242} \mathrm{Pu},{ }^{244} \mathrm{Pu},{ }^{243} \mathrm{Am},{ }^{245} \mathrm{Cm},{ }^{248} \mathrm{Cm},{ }^{249} \mathrm{Bk}$, and ${ }^{249} \mathrm{Cf}$. So far not confirmed are the electron-capture decays of the isotopes ${ }^{266} \mathrm{Db},{ }^{268} \mathrm{Db},{ }^{287} \mathrm{Fl}$, and ${ }^{290} \mathrm{Fl}$ and the subsequently populated nuclei and decay chains, respectively. In particular, an attempt was made to re-interpret an event chain originally assigned to an $\alpha$-decay chain starting at ${ }^{289} \mathrm{Fl}$ in [34] by assigning this chain to ${ }^{290} \mathrm{Fl}$ which decays by electron capture to ${ }^{290} \mathrm{Nh}$, see [35]. These isotopes are indicated with question marks. They are shown in the figure with the intention of reminding that electron capture is a likely process in the region of SHN although it is difficult to prove experimentally. The magic numbers for the protons predicted at element 114 and/or 120 are emphasized. The bold dashed lines mark proton number 108 and neutron numbers 152 and 162. Nuclei with that number of protons or neutrons have increased stability, however, they are deformed contrary to the spherical SHN. The crossing at $Z=114$ and $N=162$ reflects the uncertainty, whether nuclei in that region are deformed or spherical. The background structure shows the calculated ground-state shell-correction energy in steps of $1 \mathrm{MeV}$ from $-3 \mathrm{MeV}$ (light blue) to $-7 \mathrm{MeV}$ (dark blue) according to the macroscopic-microscopic model $[17,18]$. 
(Separator for Heavy Ion reaction Products) at GSI [47, 48], in the confirmation of part of these data and in the production of a new isotope of element 113 at GARIS (Gas-filled Recoil Ion Separator) at RIKEN [53]. New neutron-rich isotopes of element 112 and the new elements from 113 to 118 were produced at DGFRS (Dubna Gas-Filled Recoil Separator) at FLNR [52]. Isotopes which are presently known in the region of heavy and super-heavy nuclei are shown in fig. 3.

A key role in answering open questions related to the location of the major shell closure in the region of SHN plays the synthesis of isotopes of element 120. However, recent attempts using fusion reactions with targets of ${ }^{244} \mathrm{Pu}$ [54], ${ }^{238} \mathrm{U}$ [55] were negative or, as in the case of ${ }^{249} \mathrm{Cf}$ [56], the data are not yet completely analyzed.

In an attempt to produce an isotope of element 120 , the reaction ${ }^{54} \mathrm{Cr}+{ }^{248} \mathrm{Cm} \rightarrow{ }^{302} 120^{*}$ was investigated at SHIP [35]. This reaction is more asymmetric than the reactions ${ }^{64} \mathrm{Ni}+{ }^{238} \mathrm{U}$ and ${ }^{58} \mathrm{Fe}$ $+{ }^{244} \mathrm{Pu}$ and thus less Coulomb repulsion exists in the entrance channel. Although the reaction ${ }^{50} \mathrm{Ti}+$ ${ }^{249} \mathrm{Cf}$ is even more asymmetric, the choice using a ${ }^{248} \mathrm{Cm}$ target could profit from being three neutrons nearer to the $\mathrm{N}=184$ shell closure. To date, the measured cross-sections were always higher when more neutron rich projectile and/or target isotopes were used. It was planned to reach a cross-section limit of $100 \mathrm{fb}$ for which a beam time of 140 days was requested.

Safe operation of SHIP under the experimental conditions was successfully tested in a preparatory experiment in 2010 [57]. In the reaction ${ }^{48} \mathrm{Ca}+{ }^{248} \mathrm{Cm} \rightarrow{ }^{296} \mathrm{Lv}^{*}$ decay data of ${ }^{293} \mathrm{Lv}$ and ${ }^{292} \mathrm{Lv}$, previously obtained at FLNR, were confirmed.

During a first part of the ${ }^{54} \mathrm{Cr}+{ }^{248} \mathrm{Cm}$ experiment lasting 38 days in 2011, three correlated signals were measured occurring within a period of $279 \mathrm{~ms}$. The surprising properties of the signals were that the energies of the first two signals are in agreement with calculated values for the $\alpha$ energies of ${ }^{299} 120$ and its daughter isotope ${ }^{295} 118[19,58,59]$ and the third signal agrees with the previously measured $\alpha$ energy and lifetime of the granddaughter ${ }^{291} \mathrm{Lv}$ [60]. And, secondly, a very low probability was calculated that the chain of signals was produced by chance.

Nevertheless, an unambiguous assignment of the signals cannot be made. The implantation of the parent nucleus with a short lifetime corresponding to the high decay energy was not found. The time to the nearest implanted nucleus is unexpectedly long, and it cannot be distinguished from an accidental event. An unexpected long lifetime was measured also for the relatively high decay energy of the daughter nucleus. An explanation of the first two signals as isomeric decays is possible. However, without confirmation in further experiments such explanations remain speculative. Unfortunately, the experiment could not be continued. Beam time was not allocated or already allocated beam time was canceled in favor of other experiments.

\section{Perspectives}

Despite the synthesis of nuclei as heavy as ${ }^{294} 118$, the extension of the island in proton and neutron numbers and also the locations of the centers of highest stability resulting in highest production crosssections and that of longest half-lives is not yet explored. The reasons are experimental constraints like availability of targets, limited beam intensities and consequently long measuring times at crosssection levels of picobarn and below.

The progress towards the exploration of the island of SHN is difficult to predict. Hot fusion based on actinide targets and ${ }^{48} \mathrm{Ca}$ beams terminate at element 118 , because targets beyond $\mathrm{Cf}$ can be produced only with tremendous costs and efforts. How heavier beams like ${ }^{50} \mathrm{Ti}$, ${ }^{54} \mathrm{Cr}$, etc. will affect the fusion cross-section is subject of experiments planned for the near future. However, these heavier beams are mandatory for exploration of the island of SHN into the north-east direction, the direction towards new elements. Strong shell effects, if they exist at $Z=120$ or 126 , could positively influence 
the reaction cross-sections. Alpha energies measured of more isotopes of element 118 and of the new element 120 may help to settle this uncertainty.

In order to deduce information on SCE and related fission barriers, relative masses of nuclei within $\alpha$-decay chains of five neighboring even element isotopes were compared with theoretical values of the two MM models by Möller et al. [19] and Sobizcweski et al. [58, 59]. From this study less strong SCE values and consequently lower fission barriers were deduced for Fl and Lv isotopes than predicted in $[19,61]$ where particularly high fission barriers for these isotopes were calculated. The results of this study and consequences for cross-section calculations were published in [62]. There, arguments are given that the cross-section for synthesis of element 120 could be higher than previously expected. The reason for this contradictory statement, higher cross-sections at lower fission barriers, is that at lower fissions barriers for isotopes of elements $\mathrm{Fl}$ and $\mathrm{Lv}$ the estimated probability of reseparation in the entrance channel has to be reduced in order to reproduce the measured cross-sections for production of these nuclei. At a reduced probability for re-separation and less rapid decrease of the fission barriers for nuclei beyond Lv, the higher evaporation residue cross-sections can be expected. If confirmed in future experiments, the moderate decrease of fission barriers towards heavier elements might be caused by a not negligible effect of the closure of a subshell at element 120 and a low level density extending up to $\mathrm{Z}=126$.

In this context, it is worthwhile to study the transition from high to low excitation energies, which is expected to occur with actinide targets and the strongly bound isotopes in the vicinity of iron and nickel for synthesis of elements on the way to $Z=126$ (see fig. 5 in [62]). For the heaviest systems, excitation energies for fusion at beam energies just enough high for reaching a contact configuration, attain values close to the one neutron binding energy. In these cases hot fusion changes to cold fusion also for the reactions with actinide targets. Increasing losses by re-separation in the entrance channel due to increasing Coulomb repulsion at higher charge of the beam particles could possibly be compensated by a lower probability of $\mathrm{CN}$ fission.

The results presented in [35, 62] may be of interest for search experiments for element 120 being in preparation at other laboratories. At DGFRS in Dubna a target of ${ }^{249} \mathrm{Cf}(50.4 \%),{ }^{250} \mathrm{Cf}(13.5 \%)$, and ${ }^{251} \mathrm{Cf}(36.1 \%)$ will be irradiated with a ${ }^{48} \mathrm{Ca}$ beam. In this experiment heavier isotopes than the known ${ }^{294} \mathrm{Og}$ will be produced. The results will also show if the cross-section will increase when heavier target isotopes are used and the $\mathrm{CN}$ are closer to the center of strongest SCE, see figs. 2 and 3. In a second step, it is planned to switch the beam from ${ }^{48} \mathrm{Ca}$ to ${ }^{50} \mathrm{Ti}$ for synthesis of element 120 $[63,64]$. The target of mixed isotopes has to be used because highly enriched material of ${ }^{250} \mathrm{Cf}$ or ${ }^{251} \mathrm{Cf}$ is not available and ${ }^{252} \mathrm{Cf}$ is excluded due to its too high specific activity. Such a target wheel can be handled only under extreme safety conditions.

At GARIS at RIKEN, it is planned to produce isotopes of element 118 using the reaction ${ }^{50} \mathrm{Ti}+$ ${ }^{248} \mathrm{Cm} \rightarrow{ }^{298} \mathrm{Og} *$ and in a second step to switch the beam to ${ }^{54} \mathrm{Cr}$ for synthesis of element $120[65,66]$.

No technical limitations exist for exploration of the island of SHN towards the west. Sufficient neutron deficient projectile isotopes are available. However, due to Q-value effects the excitation energy of the $\mathrm{CN}$ at barrier energies will increase.

Most interesting, but also most difficult, will be the synthesis of more neutron-rich isotopes located in the south-east direction of the island of SHN. There, the longest half-lives are expected. Reactions using radioactive beams and multi-nucleon transfer reactions are options to be studied in the future. Searching for electron capture decay branches could be another option. An example, as discussed in [35], could be electron capture of ${ }^{290} \mathrm{Fl}$ populating an $\alpha$-decay chain starting at the odd-odd isotope ${ }^{290} \mathrm{Nh}$ and terminating by SF of ${ }^{278} \mathrm{Bh}$. Decay chains of odd-odd nuclei are expected to reveal particularly long lifetimes due to specific hindrance factors. It is interesting to note that EC of ${ }^{290} 114$ was already considered by E.O. Fiset and J.R. Nix in 1972 [29]. In this theoretical study it was predicted 
that ${ }^{290} 114$ will be populated by $\alpha$ decay in a decay chain starting at ${ }^{302} 120$. In a recent paper, V.I. Zagrebaev, A.V. Karpov, and Greiner also investigated the possibility of populating neutron-rich SHN via EC [67].

Using transfer reactions as suggested in [68], low beam energies and hence observation in the zero degree direction are mandatory, in order to produce the fragments at the lowest possible excitation energy and thus reduce fission, in particular of the heavy, high $\mathrm{Z}$ fragment. Systems as heavy as ${ }^{238} \mathrm{U}$ $+{ }^{248} \mathrm{Cm}$ are technically possible and could be investigated with a modern separator and detection methods. These methods will also allow for measuring contact times of dinuclear systems by making use of the kinematics of the reaction products at the moment of re-separation after rotation of the system at small impact parameters [69].

Excitation functions have to be measured, which provide information on how fast the cross-section decreases with increasing energy due to diabatic processes in the entrance channel and fission of the $\mathrm{CN}$, and how fast they decrease on the low energy side due to the fusion barrier and re-separation of projectile and target nuclei. From both slopes, information about the shape of the fission and the fusion barriers can be obtained.

The study of transfer products may also open a direct access to the first steps of the processes resulting in fusion. Due to the low beam energy the reactions occur in central collisions and the reaction partners re-separate in and opposite to the beam direction. Therefore velocity separators like SHIP are an ideal tool to study these processes.

The classical cold fusion reactions based on lead and bismuth targets will be further used for exploring the regions of increased stability of deformed heavy nuclei located around $Z=100, N=152$ and $Z=108, N=162$. How far these regions extend into the west and north-west direction is a question which has to be answered. Also, whether SF or proton emission will determine the limits.

Element 114 was discovered in a hot fusion reaction. Another possibility could be the cold fusion reaction ${ }^{76} \mathrm{Ge}+{ }^{208} \mathrm{~Pb}$. As predicted by the late Wladyslaw Świastecki using his fusion-by-diffusion model [70], the cross-section should be considerably higher than the one for synthesis of element 113. This experiment is still waiting to be performed.

At high enough cross-sections, the measurements can be complemented by in-beam $\gamma$-ray spectroscopy using recoil-decay tagging methods in order to study the influence of angular momentum on the fusion and survival probability. Observation of characteristic $\mathrm{X}$ rays emitted during the deexcitation cascade could help identifying the produced nuclei.

Similar arguments hold for the measurement of $\gamma$ rays and $\mathrm{X}$ rays emitted from the radioactive decay of the separated isotopes. Only at high enough yield the measured signals can be assigned to $\mathrm{X}$ rays on the basis of the characteristic intensity distribution which distinguishes $\mathrm{X}$ rays from $\gamma$ transitions. A recent attempt assigning decay chains measured in the reaction ${ }^{48} \mathrm{Ca}+{ }^{243} \mathrm{Am}$ to element 115 using $\alpha$-X-ray coincidence technique failed, although a relatively large amount of 30 decay chains was observed [71]. An example of a conclusive X-ray identification of element 104 is published in [72]. In that experiment a number of about $1000 \alpha$ particles were necessary in order to obtain a convincing X-ray spectrum of element 102.

Interesting in this context is a suggestion using collision induced $\mathrm{X}$ rays for element identification, measured in coincidence with particle detectors [73]. Similar as the decay X-rays, the method will not allow for identification of new elements produced at low counting rates. However, with modern arrays of X-ray detectors this method may be promising for determining the element distribution in multi-nucleon transfer reactions or in fusion reactions at inverse kinematics. In these cases the higher energy of the reaction products increases the yield of the produced $\mathrm{X}$ rays.

A comparison of experimental and theoretical SF half-lives of the known even-even isotopes of Ds and Fl is difficult, because these nuclei are located in a transitional region between deformed heavy 
nuclei and spherical SHN and the degree of deformation is not known. The measurement of small $\mathrm{SF}$ branchings of more neutron rich even isotopes of Fl, which are located closer to the center of the island of spherical SHN, will allow for a solid comparison of experimental and theoretical SF halflives. Expected are significant data on fission barriers of spherical SHN, which are needed for better estimates of production cross-sections in various reactions as $e . g$. fusion with radioactive neutron rich beams, multi-nucleon transfer reactions and rapid neutron capture in a stellar environment. The latter aspect is closely related to the question, if SHN could be produced in nature and how long they could survive.

Important for determination of masses of nuclei along $\alpha$-decay chains is the detection of small $\alpha$-decay branchings, in particular of neutron-rich even-even isotopes of Ds, which decay dominantly by SF, and $\alpha$ decay was not yet observed.

A direct measurement of the mass of one nucleus of the decay chain is necessary for determining absolute masses of the nuclei of the whole chain. Stopping of the separated reaction products in gas catchers and injection of the low energy beam in multi-reflection time-of-flight mass spectrometers (MR-TOF) [74] or Penning trap mass spectrometers [75] will allow for an accurate mass determination. Neutron rich nuclei being located in the region of elements from $\mathrm{Rf}$ to $\mathrm{Sg}$ are presently produced at low cross-section as decay products of SHN. However, in future experiments with radioactive beams or in multi-nucleon transfer reactions these nuclei could be directly produced with higher yield. The long half-lives expected for some of the neutron-rich heavy nuclei, are not a limitation, but on the contrary, the precision of the measurement increases with increasing half-life. The mass measurements of No and Lr isotopes with SHIPTRAP represents a convincing example for the application of the method also in the region of heavy nuclei [75].

Stopping of the separated reaction products in gas catchers and injection of the low energy beam in MR-TOF or Penning-trap spectrometers will also allow for isobaric purification and precise decay spectroscopy. In addition, atomic beam experiments as e.g. collinear laser spectroscopy and SternGerlach experiments will also become possible. In the near future, further technical improvements such as a cryogenic stopping cell, will be implemented, a major step into direction of higher yield.

The region far beyond element 126 was already addressed theoretically 40 years ago. For example, a region of relatively higher stability against $\mathrm{SF}$ was predicted at $\mathrm{Z}=164$ and $\mathrm{N}=318$ in [28]. Although such heavy nuclei may not exist, the shell structure could influence the lifetime of an intermediate resonance like structure.

The properties of toroidal and spherical bubble nuclei were presented in [76, 77]. A fullerene-type structure consisting of $\alpha$ clusters was suggested for ${ }^{304} 120$ in [78]. Although speculative, the highly advanced experimental technology should be used also for some experiments to search for such really exotic phenomena in the region of SHN and beyond, which is accessible using the heaviest beams and targets.

Accelerators, separators, detectors, signal processing, and data acquisition presently in use are highly developed and should be used for the continuation of experiments. However, improvements are still possible. An overview on current developments is given in a contribution to this conference by G. Münzenberg [79]. At expected higher beam intensities, targets must be developed, which are not immediately destroyed. The efficiency and resolution of the separators can be further increased and the background reduced. Detector shuttles could be used for distinguishing nuclei with short and long half-lives. Secondary experiments could be installed if the valuable beam is not stop inside the separator but directed to another target.

The most powerful dedicated facility under construction will be the 'SHE Factory' at FLNR in Dubna delivering beam intensities of up to 10 particle $\mu \mathrm{A}$. The GANIL laboratory in France will open new facilities to study SHN. The new Facility for Radioactive Ion Beams (FRIB), under development 
at Michigan State University, intends to use RIBs to study more neutron-rich SHN. At GSI, a new accelerator dedicated to SHN research was already suggested in 1999 [80]. The advantages of a superconducting CW (continuous wave) linear accelerator were worked out in 2004 and presented in $[81,82]$. A factor of three less power consumption was estimated while the beam intensity is increased by a factor of 3.8 even without further increase of the beam intensity from the ion source, compared to the performance of the present UNILAC.

New and more precise experimental data will again trigger theoretical studies. So, one can hope that stability of SHN and the various reactions for producing them will be better understood in the future.

\section{Acknowledgements}

In deep sadness we received the news of the decease of Professor Walter Greiner on October 6th, 2016. For many of us Walter was mentor, colleague, and friend. Walter was one of the fathers of GSI founded in 1969. This farsighted initiative led to the discovery of the new elements from bohrium ( $Z=107)$ to copernicium (112). Walter considered the study of super-heavy nuclei and elements as an opportunity to extend nuclear and atomic physics and chemistry far into the unknown resulting in new insights into the mysteries of nature. His ideas of 'cold fusion valleys' and fragmentation theory have successfully driven experimental search for these super-heavy nuclei.

I am particularly grateful for his continuous support especially in times of reduced resources and pessimistic assessments. With gratitude I remember the extensive discussions with him during taxi rides from Sheremetyevo airport to Dubna and back when we visited the program advisory committee at FLNR, which he led as chairman for many years.

I am also deeply obliged to my colleagues at GSI and the former SHIP group and participating people from other laboratories or institutes, with whom I could spend a most exciting and challenging time doing experiments with heavy ions.

The current article reproduces some text from an article published in J. Phys. G: Nucl. Part. Phys. 42, 114001 (2015). That article provides a wider comparison of experimental work on cold and hot fusion reactions based on targets of ${ }^{208} \mathrm{~Pb},{ }^{209} \mathrm{Bi}$ and isotopes of actinides, respectively.

\section{References}

[1] E. Fermi, Nature 133, 898 (1934)

[2] O. Hahn, L. Meitner, F. Strassmann Naturwissenschaften 23, 544 (1935)

[3] O. Hahn, F. Straßmann, Naturwissenschaften 27, 11 (1939)

[4] L. Meitner, O.R. Frisch, Nature 143, 239 (1939)

[5] G.N. Flerov, K.A. Petrjak, Phys. Rev. 58, 89 (1940)

[6] G. Fea, Nuovo Cimento 12, 368 (1935)

[7] J. Magill, G. Pfenning, R. Dreher, Z. Sóti, Nucleonica GmbH, 76344 EggensteinLeopoldshafen, Germany (2015)

[8] J.A. Wheeler, Niels Bohr and the Development of Physics, (Pergamon Press, London, 1955)

[9] J.A. Wheeler, Proc. Int. Conf. on the Peaceful Uses of Atomic Energy, Geneva, 1955, (United Nations, New York, 1955)

[10] G. Werner, J.A. Wheeler, Phys. Rev. 109, 126 (1958)

[11] G. Soff, J. Rafelski, W. Greiner, Phys. Rev. A 7, 903 (1973)

[12] J. Reinhardt, B. Müller, W. Greiner, Phys. Rev. A 24, 103 (1981)

[13] C. Kozhuharov, P. Kienle, E. Berdermann, H. Bokemeyer, J. S. Greenberg, Y. Nakayama, P. Vincent, H. Backe, L. Handschug, E. Kankeleit, Phys. Rev. Lett. 42, 376 (1979) 
[14] M. Göppert-Mayer, Phys. Rev. 74, 235 (1948)

[15] O. Haxel, J.H.D. Jensen, H.E. Suess, Die Naturwissenschaften 36, 376 (1949)

[16] A. Sobiczewski, F.A. Gareev, B.N. Kalinkin, Phys. Lett. 22, 500 (1966)

[17] R. Smolanczuk, J. Skalski, A. Sobiczewski, Phys. Rev. C 52, 1871 (1995)

[18] R. Smolanczuk, A. Sobiczewski, Proc. XV. Nuclear Physics Divisional Conference on Low Energy Nuclear Dynamics, St.Petersburg, Russia, 1995, edited by Yu.Ts. Oganessian, R. Kalpakchieva, W. von Oertzen, (World Scientific, Singapore, 1995)

[19] P. Möller, J.R. Nix, K.L. Kratz, At. Data Nucl. Data Tables 66, 131 (1997)

[20] V.M. Strutinsky, Nucl. Phys. A 95, 420 (1967)

[21] W.D. Myers, W.J. Świątecki, Nucl. Phys., 81, 1 (1966)

[22] A. Sobiczewski, F.A. Gareev, B.N. Kalinkin, Phys. Lett. 22, 500 (1966)

[23] H. Meldner, Ark. Fys. 36, 593 (1967)

[24] S.G. Nilsson, J.R. Nix, A. Sobiczewski, Z. Szymanski, S. Wycech, C. Gustafson, P Möller, Nucl. Phys. A 115, 545 (1968)

[25] S.G. Nilsson, S.G. Thompson, C.F. Tsang, Phys. Lett. B 28, 458 (1969)

[26] S.G. Nilsson, C.F. Tsang, A. Sobiczewski, Z. Szymanski, S. Wycech, C. Gustafson, I.L. Lamm, P. Möller, B. Nilsson, Nucl. Phys. A 131, 1 (1969)

[27] U. Mosel, W. Greiner, Z. Phys. 222, 261 (1969)

[28] J. Grumann, U. Mosel, B. Fink, W. Greiner, Z. Phys. 228, 371 (1969)

[29] E.O. Fiset, J.R. Nix, Nucl. Phys. A, 193, 647 (1972)

[30] A. Sobiczewski, Physica Scripta A 10, 47 (1974)

[31] J. Randrup, S.E. Larsson, P. Möller, S.G. Nilsson, K. Pomorski, A. Sobiczewski, Phys. Rev. C 13, 229 (1976)

[32] S.M. Polikanov, V.A. Druin, V.A. Karnaukov, V.L. Mikheev, A.A. Pleve, N.K. Skobolev, V.G. Subotin, G.M. Ter-Akopian, V.A. Fomichev, Sov. Phys. JETP 15, 1016 (1962)

[33] Yu.Ts. Oganessian, A.G. Demin, A.S. Iljinov, S.P. Tretyakova, A.A. Pleve, Yu.E. Penionzhkevich, M.P. Ivanov, Yu.P. Tretyakov, Nucl. Phys. A 239, 157 (1975)

[34] Yu.Ts. Oganessian, V.K. Utyonkov, Yu.V. Lobanov, F.Sh. Abdullin, A.N. Polyakov, I.V. Shirokovsky, Yu.S. Tsyganov, G.G. Gulbekian, S.L. Bogomolov, B.N. Gikal, et al., Phys. Rev. Lett. 83, 3154, (1999)

[35] S. Hofmann, S. Heinz, R. Mann, J. Maurer, G. Münzenberg, S. Antalic, W. Barth, H.G. Burkhard, L. Dahl, K. Eberhardt et al., Eur. Phys. J. A, 52, 180 (2016)

[36] W. Grimm, G. Herrmann, H.-D. Schüssler, Phys. Rev. Lett. 26, 1040 (1971)

[37] R.V. Gentry, T.A. Cahill, N.R. Fletcher, H.C. Kaufmann, X.R. Medsker, J.W. Nelson, R.G. Flocchini, Phys. Rev. Lett. 3711 (1976)

[38] G.N. Flerov, G.M. Ter-Akopian, Rep. Prog. Phys. 46, 817 (1983)

[39] G.M. Ter-Akopian, S.N. Dmitriev, Nucl. Phys. A 944, 177 (2015)

[40] S. Ćwiok, J. Dobaczewski, P.H. Heenen, P. Magierski, W. Nazarewicz, Nucl. Phys. A, 611, 211 (1996)

[41] K. Rutz, M. Bender, T. Bürvenich, T. Schilling, P.G. Reinhard, J.A. Maruhn, W. Greiner, Phys. Rev. C 56, 238 (1997)

[42] A.T. Kruppa, A.T. Kruppa, M. Bender, W. Nazarewicz, P.G. Reinhard, T. Vertse, S. Ćwiok, Phys. Rev. C61, 034313 (2000)

[43] M. Bender, W. Nazarewicz, P.G. Reinhard, Phys. Lett. B 515, 42 (2001)

[44] M. Bender, P. Bonche, T. Duguet, P.H. Heenen, Nucl. Phys. A 723, 354 (2003) 
[45] S. Schramm, Phys. Rev. C 66, 064310 (2002) and private communication (2014)

[46] D.C. Hoffman, Nucl. Phys., A 502, 21c (1989)

[47] G. Münzenberg, Rep. Prog. Phys. 51, 57 (1988)

[48] S. Hofmann, G. Münzenberg, Rev. Mod. Phys. 72, 733 (2000)

[49] G. Münzenberg, In-flight separation of heavy ion beams in Experimental Techniques in Nuclear Physics, edited by D.N. Poenaru, W. Greiner, (Walter de Gruyter, Berlin, New York, 1997)

[50] S. Hofmann, Rep. Prog. Phys. 61, 639 (1998)

[51] K.E. Gregorich, Nucl. Instrum. Methods Phys. Res., Sect. A 711, 47 (2013)

[52] Yu.Ts. Oganessian, V.K. Utyonkov, Rep. Prog. Phys. 78, 036301 (2015)

[53] K. Morita, Nucl. Phys. A 944, 30 (2015)

[54] Yu.Ts. Oganessian, V.K. Utyonkov, Yu.V. Lobanov, F.Sh. Abdullin, A.N. Polyakov, R.N. Sagaidak, I.V. Shirokovsky, Yu.S. Tsyganov, A.A. Voinov, A.N. Mezentsev et al., Phys. Rev. C 79, 024603 (2009)

[55] S. Hofmann, D. Ackermann, S. Antalic, V.F. Comas, S. Heinz, J.A. Heredia, F.P. Heßberger, J. Khuyagbaatar, B. Kindler, I. Kojouharov et al., GSI Scientific Report 2008, GSI Report 2009-1, $131(2009)$

[56] J. Khuyagbaatar, A. Yakushev, Ch.E. Düllmann, H. Nitsche, J. Roberto, D. Ackermann, L.-L. Andersson, M. Asai, H. Brand, M. Block et al., GSI Scientific Report 2012, GSI Report 2013-1, $131(2013)$

[57] S. Hofmann, S. Heinz, R. Mann, J. Maurer, J. Khuyagbaatar, D. Ackermann, S. Antalic, W. Barth, M. Block, H.G. Burkhard et al., Eur. Phys. J. A 48, 62 (2012)

[58] I. Muntian, S. Hofmann, Z. Patyk, A. Sobiczewski, Acta Phys. Pol. B, 34, 2073 (2003) and A. Sobiczewski, private communication (2014)

[59] I. Muntian, Z. Patyk, A. Sobiczewski, Phys. At. Nucl., 66, 1015 (2003)

[60] Yu.Ts. Oganessian, V.K. Utyonkov, Yu.V. Lobanov, F.Sh. Abdullin, A.N. Polyakov, I.V. Shirokovsky, Yu.S. Tsyganov, G.G. Gulbekian, S.L Bogomolov, B.N. Gikal et al., Phys. Rev. C69, 054607 (2004)

[61] P. Möller, A.J. Sierk, T. Ichikawa, A. Iwamoto, M. Mumpower, Phys. Rev. C, 91, 024310 (2015)

[62] S. Hofmann, S. Heinz, R. Mann, J. Maurer, G. Münzenberg, S. Antalic, W. Barth, L. Dahl, K. Eberhardt, R. Grzywacz et al., Eur. Phys. J. A 52, 116 (2016)

[63] J.H. Hamilton, S. Hofmann, Y.T. Oganessian, Annu. Rev. Nucl. Part. Sci. 63, 383 (2013)

[64] V.K. Utyonkov, Int. Symposium 'Super Heavy Nuclei', College Station, Texas, USA, 2015, http://cyclotron.tamu.edu/she2015/assets/pdfs/presentations/Utyonkov_SHE_2015_TAMU.pdf

[65] K. Morita, et al., RIKEN Accel. Prog. Rep. 2015, 49, 385 (2016)

[66] K. Morita, Int. Symposium 'Super Heavy Nuclei', College Station, Texas, USA. 2015, http://cyclotron.tamu.edu/she2015/assets/pdfs/ presentations/Morita_SHE_2015_TAMU.pdf

[67] V.I. Zagrebaev, A.V. Karpov, W. Greiner, Phys. Rev. C, 85, 014608 (2012)

[68] V.I. Zagrebaev, Yu.Ts. Oganessian, M.G. Itkis, W. Greiner, Phys. Rev. C 73, 31602(R) (2006)

[69] S. Heinz, V. Comas, F.P. Heßberger, S. Hofmann, D. Ackermann, H.G. Burkhard, Z. Gan, J. Heredia, J. Khuyagbaatar, B. Kindler et al., Eur. Phys. J. A 38, 227 (2008)

[70] W.J. Świątecki, K. Siwek-Wilczyńska, J. Wilczyński, Phys. Rev. C 71, 014602 (2005)

[71] D. Rudolph, U. Forsberg, P. Golubev, L.G. Sarmiento, A. Yakushev, L.L. Andersson, A. Di Nitto, Ch.E. Duüllmann, J.M. Gates, K.E. Gregorich et al., Phys. Rev. Lett. 111, 112502 (2013) 
[72] C.E. Bemis, Jr., R.J. Silva, D.C. Hensley, O.L. Keller, Jr., J.R. Tarrant, L.D. Hunt, P.F. Dittner, R.L. Hahn, C.D. Goodman, Phys. Rev. Lett. 31, 647 (1973)

[73] W. Faust, P. Armbruster, S. Hofmann, G. Münzenberg, H. Ewald, K. Güttner, J. Radioanal. Nucl. Chem. 55175 (1980)

[74] S. Heinz, W. Barth, B. Franczak, H. Geissel, M. Gupta, S. Hofmann, S. Mickat, G. Münzenberg, W.R. Plaß, C. Scheidenberger, H. Weick, M. Winkler, Nucl. Instrum. Methods Phys. Res. B 317, 354 (2013)

[75] M. Block, D. Ackermann, K. Blaum, C. Droese, M. Dworschak, S. Eliseev, T. Fleckenstein, E. Haettner, F. Herfurth, F.P. Heßberger, Nature 463, 785 (2010)

[76] C.Y. Wong, Ann. Phys. 77, 279 (1973)

[77] K. Dietrich, K. Pomorski, Phys. Rev. Lett. 80, 37 (1998)

[78] W. Greiner, Int. J. Mod. Phys. E 17, 2379 (2008)

[79] G. Münzenberg, Super Heavy Elements - Experimental Developments, contribution to this conference

[80] S. Hofmann, SHIP-2000 - A proposal for the study of superheavy elements, (GSI-Report 99-02, 1999)

[81] S. Hofmann, D. Ackermann, W. Barth, L. Dahl, F.P. Heßberger, B. Kindler, B. Lommel, R. Mann, G. Münzenberg, K. Tinschert, U. Ratzinger, A. Schempp, International Symposium on Exotic Nuclei, EXON-2004 (World Scientific Publishing, Singapore, 2005)

[82] W. Barth, Int. Symposium 'Super Heavy Nuclei', College Station, Texas, USA, 2015, http://cyclotron.tamu.edu/she2015/assets/pdfs/ presentations/Barth_SHE_2015_TAMU.pdf 